\title{
Mapping Information to Audio and Tactile Icons
}

\author{
Eve Hoggan ${ }^{1,2}$, Roope Raisamo ${ }^{2}$ and Stephen Brewster ${ }^{1}$ \\ ${ }^{1}$ Glasgow Interactive Systems Group \\ University of Glasgow, Glasgow, G12 8QQ, UK \\ ${ }^{2}$ Tampere Unit for Computer-Human Interaction \\ \{eve, stephen\}@dcs.gla.ac.uk \\ University of Tampere, FIN-33014 Tampere, Finland \\ rr@cs.uta.fi
}

\begin{abstract}
We report the results of a study focusing on the meanings that can be conveyed by audio and tactile icons. Our research considers the following question: how can audio and tactile icons be designed to optimise congruence between crossmodal feedback and the type of information this feedback is intended to convey? For example, if we have a set of system warnings, confirmations, progress updates and errors: what audio and tactile representations best match the information or type of message? Is one modality more appropriate at presenting certain types of information than the other modality? The results of this study indicate that certain parameters of the audio and tactile modalities such as rhythm, texture and tempo play an important role in the creation of congruent sets of feedback when given a specific type of information to transmit. We argue that a combination of audio or tactile parameters derived from our results allows the same type of information to be derived through touch and sound with an intuitive match to the content of the message.
\end{abstract}

\section{Categories and Subject Descriptors}

H.5.2 [User Interfaces]: Haptic I/O, Auditory (non-speech) feedback, Style guides.

\section{General Terms}

Human Factors, Design.

\section{Keywords}

Mobile touchscreen interaction, audio interaction, tactile interaction, information mapping, Earcons, Tactons.

\section{INTRODUCTION}

Finding alternative forms of information presentation for mobile device interfaces is fundamental given that we spend much of our daily lives using mobile devices. There are a large variety of different applications available for mobile devices and in turn, this means that there is a large variety of information that needs to be presented. The majority of this is displayed visually on extremely small screens. This places a high demand on the visual sense and explains the extent to which users can often spend more time focused on the screen than on the environment or task in hand.

Permission to make digital or hard copies of all or part of this work for personal or classroom use is granted without fee provided that copies are not made or distributed for profit or commercial advantage and that copies bear this notice and the full citation on the first page. To copy otherwise, or republish, to post on servers or to redistribute to lists, requires prior specific permission and/or a fee.

ICMI-MLMI'09, November 2-4, 2009, Cambridge, MA, USA.

Copyright 2009 ACM 978-1-60558-772-1/09/11...\$10.00.
Many commercial devices employ the use of audio and tactile feedback to provide simple alerts, such as incoming call notifications, through the use of ringtones and vibrations. The possibilities of communicating information and enhancing interaction through senses other than vision such as sound and touch has generated a rich body of research. Numerous user studies have explored methods of encoding information in each modality through the use of icons such as Auditory Icons [8], Earcons [3], Tactons [4] and Haptic Icons [14]. Results of experiments using each of these types of icon have shown that high recognition rates can be achieved with a small amount of training. Alongside this research, there have been several studies exploring the effects on user performance and satisfaction of adding audio and tactile feedback to mobile applications $[5,12]$. These studies have shown that both audio and tactile feedback can improve user performance with mobile device applications over visual feedback alone.

Despite this rich body of work, there are few guidelines on how to encode specific information using the different modalities. Although it has been shown that, for instance, rhythm is an effective audio and tactile parameter, the mapping of information to this parameter in experiments is usually random. For example, in Brown et al. [4] Tactons were designed to represent calendar alerts. However, this may not be the best type of information to represent with those particular Tactons. The question now is how to make use of this research to provide an alternative to all of the different types of visual information provided by different mobile applications. Therefore, this paper discusses the possible meanings that can be conveyed by Earcons [3] and Tactons [4]. How can audio and tactile icons be designed to optimise congruence between crossmodal feedback and the type of information this feedback is intended to convey? Congruence is a relationship between objects that implies agreement, harmony, conformity or correspondence (American Heritage Dictionary). In terms of this research, we define congruence as an intuitive match or harmony between the designs of feedback from different modalities and information types. For example, if we have a set of system warnings, confirmations, progress updates and errors: what audio and tactile representations best match the information or type of message? Is one modality more appropriate at presenting certain types of information than the other (e.g. [10])?

In an effort to address these questions, this paper presents the results from an experiment investigating methods of designing crossmodal audio and tactile feedback by manipulating the different parameters of each modality in order to produce congruent sets of feedback when given a specific type of information to transmit. For instance, one could imagine that an urgent warning would result in a tactile square wave with increasing intensity or, in audio, a rough timbre with increasing amplitude. Perhaps all warnings should be presented through the audio modality while all confirmations should be tactile. This experiment examined the 
relationship between 4 different information types: confirmations, errors, warnings and progress updates, and 15 sets of Earcons and Tactons. The sounds and vibrations varied in their tempo, duration, texture, spatial location and rhythm. We present practical implementation details and guidelines based on the results from our information mapping experiment focusing on the appropriateness of the audio and tactile modalities for different types of information presentation and then, more specifically, on individual design parameters. Drawing on the results, we argue that a combination of audio or tactile parameters can be produced which allow the same type of information to be derived through touch and sound with an intuitive match to the content of the message.

\section{RELATED WORK}

This study makes use of Earcons [3] and Tactons [4] for the underlying design of the crossmodal feedback. Both types of icon have been subject to a great deal of research and can provide an alternative to visual icons. Two main types of non-speech audio cues exist: Auditory Icons [8] and Earcons [2]. Auditory Icons are natural, everyday sounds used to represent events or items within a computer interface. The sounds that are used are semantically linked to the things they represent, and the meanings should therefore be easy to learn and remember. This approach is similar to a pictorial approach. Earcons, on the other hand, are structured, abstract non-speech audio messages which use an approach like the coded approach in the vibrotactile domain. Earcons use musical, rather than natural, sounds and use an abstract mapping that must be learned, as there is no semantic link between the sounds and the data they represent.

Rovers and Essen [20] mention the use of icons with haptic feedback stating that the message can be designed as a signal that can be recognised in the real world, e.g. a heartbeat, or as an abstract message based on common rules, e.g. symbols, 3 ticks for off, and variability can be represented in glyphs, e.g. changing intensity based on running speed. Tactons [4] are used as the vibrotactile counterparts of Earcons in the design of crossmodal icons. These are structured vibrotactile messages which can be used to communicate information non-visually. Tactons are created by manipulating the parameters or dimensions of cutaneous perception to encode information, such as texture, rhythm and intensity.

Although Earcons and Tactons are symbolic and it is not assumed that their meanings can be understood without training, ideally the mapping between meaning and icon should be as simple as possible and consistently interpreted in the same way among all users. At present, most design methods for audio and tactile feedback in multimodal or crossmodal applications are based on empirical knowledge, often resulting in Earcons and Tactons derived from random selection or the personal preferences of the designer. For example, Enriquez, MacLean, and Chita [7] created a set of nine haptic icons that varied in terms of waveform and frequency. They then trained participants to associate each haptic icon with an arbitrary concept, such as the name of a fruit. The next logical step would be to identify the most appropriate types of information that can be represented by these icons. Earcons and Tactons have been used to represent various different types of information in many experiments including calendar alerts [4], fairground rides [15], user interface widgets [19] and navigation alerts [22]. These information types were chosen by the designers and were perfectly appropriate for the studies in which they were used but perhaps these icons are more suited to different types of information mapping.
This research is closely related to Bernsen's concept of Modality Theory [1] which also addresses the mapping of information to different modalities. Modality Theory was introduced to concentrate on the general problem of mapping task domain information into multimodal interfaces. The outcomes of this research include a generative taxonomy of output representations and a methodology for information mapping. The methodology focuses on output modalities only whereas the research in this paper could be seen as an extension of this, investigating individual parameters within each modality.

There have been some previous studies into parameters and the meaning of symbolic sounds in the area of auditory alarm applications. Patterson [18] states that warnings should be composed using a distinctive temporal pattern to minimise the probability of confusion among different warnings. Both, rhythm and the tempo or speed of the signals also have a significant effect on the perceived urgency of the alarm [6]. Acoustically more urgent warnings produce faster responses to important, hazardous situations and events than those that are less acoustically urgent [6]. Signals with no inter-pulse intervals are rated most urgent of all. Other factors affecting perceived urgency suggested are speed and predictability of the structure of the sound [6]. There have also been many studies investigating the potential use of tactile warning signals with information displays in applied interface environments such as automobiles, cockpits and many navigation applications (e.g. [23] ).

Using a more general approach, Palomäki [17] conducted an experiment to examine the relationship between twenty-six adjectives and nine simple rhythmic sounds. The sounds varied in tempo, number of beats and rate of predictability. The study showed that some adjectives like excited, active, intense and fast, were more often associated with fast tempo rhythms and some adjectives like easy, calm, safe and slow, with slow tempo rhythms.

These studies indicate that there are many different ways to encode information in the audio and tactile modalities. Earlier research into the presentation of warnings suggests that there are specific parameters in the audio and tactile modalities that contribute to the perception of urgency in these warnings. Therefore, certain combinations of parameters in audio and tactile feedback may be more congruent than others with specific information mappings.

The approach used in this research focuses on a form of crossmodal interaction. In contrast to multimodal interaction, crossmodal interaction uses the different senses to provide the same information [10]. Within the audio and tactile modalities, it is hoped that the different parameters may be manipulated to create congruent sets of feedback to match different types of information (confirmations, progress updates, warnings and errors). Both modalities share temporal and spatial properties so the potential parameters are intensity, rate, texture, rhythmic structure, duration and spatial location. These parameters are amodal i.e. they can specify similar information across modalities [13].

\section{EXPERIMENT DESIGN}

\subsection{Stimuli}

In this experiment, two EAI C2 Tactors [16] from Engineering Acoustics were chosen to present the different sets of vibrotactile feedback (Figure 1). The audio feedback was presented through headphones attached to a Nokia N810 Internet touchscreen tablet 
(europe.nokia.com/nseries/). The volume and intensity levels of both the audio and tactile feedback were kept constant for all participants.

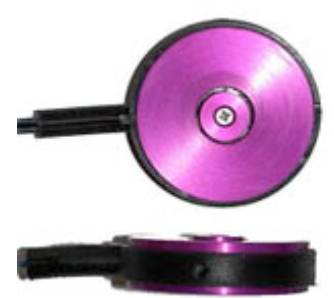

Figure 1: EAI C2 vibrotactile actuator from Engineering Acoustics.

Five different parameters were used in the design of the crossmodal Earcons and Tactons (all with a base frequency of $250 \mathrm{~Hz}$ ) and are detailed below. A baseline stimulus was created for each modality. This consisted of a one-beat smooth rhythm in a central spatial location with a duration of $500 \mathrm{~ms}$ and 60 beats per minute. This was used to provide participants with something to compare to when presented with one of the crossmodal cues from the stimulus set.

Rhythm: three different rhythms were used in the Earcons and Tactons (Figure 2). These rhythms have already been used successfully in crossmodal experiments [4, 10]. Each rhythm was made up of a different number of beats, with the 2-beat rhythm consisting of one short beat and one long beat, the 4-beat rhythm consisting of two long beats and two short beats, and the 6-beat rhythm consisting of one long beat, three short beats, and two long beats. Using a different number of beats in each rhythm helps to make the rhythms distinguishable. All rhythms lasted 500ms.

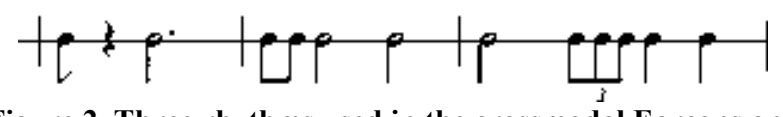

Figure 2. Three rhythms used in the crossmodal Earcons and Tactons.

Texture: three different textures were created using different waveforms in the tactile feedback and different timbres in the audio feedback. These textures have been successfully established as efficient crossmodal parameters in previous work [11]. The tactile textures were created using sine, sawtooth and square waves while the audio textures were created using a piano, tremolo cello and vibraphone timbres.

Spatial Location: three sets of spatial location patterns were used - left to right, central and circular (left to center to right to center). In terms of the tactile feedback, these alerts were presented using two C2 Tactors attached to the back of the Internet tablet. The audio crossmodal icons used three locations in a $3 \mathrm{D}$ audio soundscape to encode the information about the sender of the message sounds were placed on a horizontal plane around the user's head.

Duration: based on previous work by Geldard [9] which suggests that the minimum distinguishable difference between durations is $10 \mathrm{~ms}$ and that stimuli should not be shorter than 0.1 seconds or longer than 2 seconds, three different durations were chosen: $500 \mathrm{~ms}, 1$ second and 2 seconds. This meant that when using a duration of 2 seconds, each rhythm described above was played 4 times.
Tempo/Rate: using the guidelines provided by Geldard [9], three different rates or tempo were chosen. It is stated that there should be a minimum difference of 73 pulses per second and that users can distinguish between 8 and 10 levels so both the audio and tactile feedback used rates with intervals of $60,150,240$ beats per minute. As mentioned, the rhythm and duration of the baseline stimulus was fixed to the one-beat rhythm lasting $500 \mathrm{~ms}$ but repetition was not. So for each different tempo, the baseline one-beat rhythm was repeated at either 60,150 or 240 beats per minute over the duration of $500 \mathrm{~ms}$.

\subsection{Contexts of Use}

The majority of information provided by mobile devices and in general, any computer application can be divided into four categories: confirmations, progress updates, warnings and errors [21]. However, in different applications, a warning for example can have completely different implications. A warning from a mobile phone about battery levels is obviously not as serious as a warning from a medical monitoring system about a low heart rate. So, in a completely different use case scenario, the information and choice of crossmodal feedback design could be completely different. Then again, warnings from all different types of systems could be congruent with the same feedback.

Therefore, in this study, it was necessary to frame the experiment in different contexts. The experiment involved three different use case scenarios: everyday mobile phone, an exercise application and a medical health monitoring application. The idea being that once a congruent audio/tactile mobile phone warning, medical health warning and exercise warning have been chosen, we can extract the common parameters from each to establish the necessary components for any type of general warning information.

\subsection{Methodology}

A 5AFC (5 Alternatives Forced Choice) method was used where a range of 5 options were compared, in this case there were 5 different crossmodal cues. In the first part of the experiment, before moving on to the stimuli sets that made use of the different parameters mentioned above, experiment participants were shown a visual representation of each type of information and then presented with three choices: audio, tactile or combined audio/tactile. In this part of the experiment there were 24 tasks (4 information types $\mathrm{x} 3$ different versions presented twice). The aim of this part of the experiment was to establish whether certain modalities were preferred over others for each type of information.

Overall there were 180 tasks in the second part of the experiment using the 4 different information types and a set of 15 crossmodal cues ( 5 different parameters x 3 parameter values (e.g. rhythm 1, 2 or 3 ) repeated three times. The modality of the crossmodal cues presented was determined by the participant's answers to the first part of the experiment. For example, if audio was chosen as the most appropriate modality for confirmations, the set of 15 audio cues were presented when the task involved confirmation information types.

Participants were shown the visual representation of the information or message and then presented with five random sets of feedback using one of the subsets of the crossmodal cues discussed earlier. These cues could be compared to the baseline cue at any time. Participants were asked to pick which set of feedback matched the information best (as shown in Figure 3). 


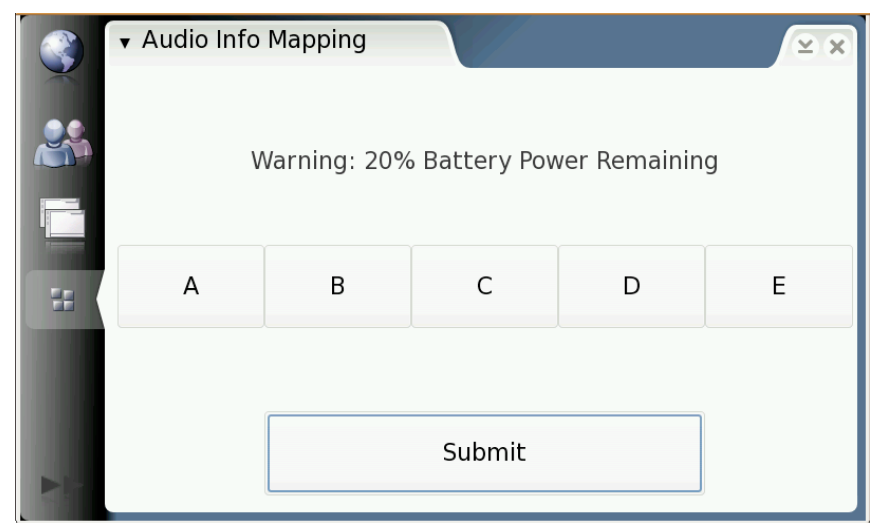

Figure 3: Screenshot of experiment interface.

Overall, eighteen participants took part in the experiment: 10 male and 8 female, all students or staff at the University of Tampere. In general terms, the experiment involved a between subjects design where three groups of six participants completed the forced choice tasks each under a different use case scenario. Examples of the different types of information for the use case scenarios are shown in Table 1. There were also two within subjects factors (modality and information type). All tasks were counterbalanced.

So in an example task, the participants were presented with a confirmation (e.g. "Email Received"). Once they were familiar with the meaning of the confirmation, they were presented with the visual version plus five random different types of audio or tactile or audio/tactile cues and asked to select which one of the five they believed were the best match for the confirmation.

Table 1: Examples of information types presented to participants during the experiment for each use case.

\begin{tabular}{|c|c|c|c|}
\hline $\begin{array}{c}\text { Information } \\
\text { Type }\end{array}$ & $\begin{array}{c}\text { Basic Mobile } \\
\text { Device Version }\end{array}$ & $\begin{array}{c}\text { Medical Health } \\
\text { Monitoring } \\
\text { Version }\end{array}$ & $\begin{array}{c}\text { Exercise Trainer } \\
\text { Version }\end{array}$ \\
\hline Confirmations & SMS Received & $\begin{array}{c}\text { Blood Pressure } \\
\text { OK }\end{array}$ & $\begin{array}{c}\text { Target Speed } \\
\text { Reached }\end{array}$ \\
\hline Errors & $\begin{array}{c}\text { Incorrect Pin } \\
\text { Code }\end{array}$ & $\begin{array}{c}\text { No Battery } \\
\text { Power }\end{array}$ & $\begin{array}{c}\text { No Movement } \\
\text { Detected }\end{array}$ \\
\hline $\begin{array}{c}\text { Progress Up- } \\
\text { dates }\end{array}$ & $\begin{array}{c}\text { Download - } \\
75 \% \text { Complete }\end{array}$ & $\begin{array}{c}\text { Blood Pressure } \\
\text { Reading in } \\
\text { Progress }\end{array}$ & $\begin{array}{c}\text { Approaching } \\
\text { Heart Rate Zone } \\
1\end{array}$ \\
\hline Warnings & Low Battery & $\begin{array}{c}\text { Low Blood } \\
\text { Pressure }\end{array}$ & High Heart Rate \\
\hline
\end{tabular}

\subsubsection{Hypotheses}

The hypotheses for this experiment were as follows:

1. Participants will consistently map specific audio and tactile cues to different types of information;

2. Different modalities will be chosen for different information types.

\section{RESULTS}

During the experiment, the experimental software recorded data on the participants' rankings of each stimulus (the number of times each audio, tactile or combined audio/tactile feedback set was chosen as the preferred match to the given information type).

\subsection{Modality}

The total number of votes for each modality when given an information type is shown in Figure 4. In terms of Confirmations, presentation using the Tactile modality received 90 votes, then Combined audio/tactile presentation received 72 votes and lastly presentation through Audio resulted in 54 votes. Combined audio/tactile feedback received the most votes for error presentation (90 votes) while tactile feedback received 66 and audio received 60 votes. For progress updates, tactile feedback received the highest number of votes (90) followed by combined audio/tactile feedback with 78 and then audio feedback with 48 votes. Lastly, combined audio/tactile presentation of warnings resulted in 96 votes followed by tactile presentation with 78 and audio with 42 .

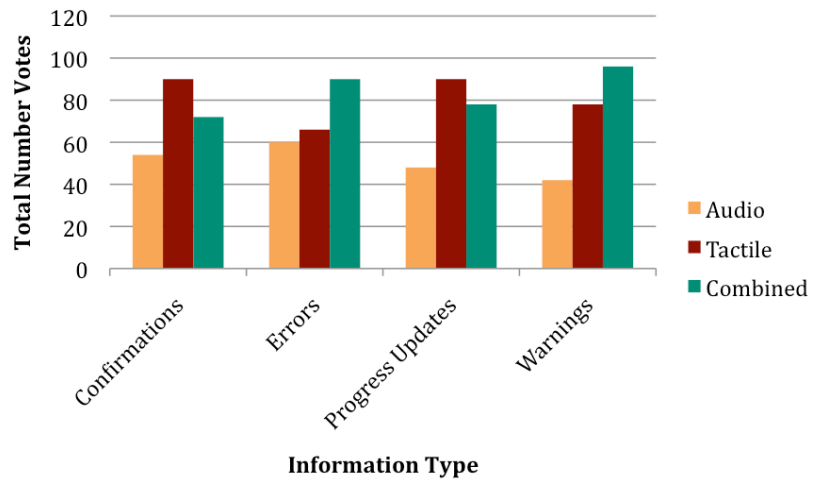

Figure 4: Total number of votes for each modality $(\max 216)$.

A Friedman test was performed on the total number of votes, comparing the effects of modality (Audio, Tactile and Combined audio/tactile) with four information types (Confirmations, Errors, Progress updates and Warnings). The Friedman Test showed a significant effect for modality $(\mathrm{Q}=36.8, \mathrm{df}=2, \mathrm{p}<0.001)$ and for information type $(\mathrm{Q}=24.57, \mathrm{df}=3, \mathrm{p}<0.001)$. Using post hoc Wilcoxon signed-rank tests, it can be seen that a significantly higher number of votes occurred for Tactile and Combined audio/tactile feedback than Audio feedback alone (with a Bonferroni corrected significance level of $\mathrm{p} \leq 0.001$ ). There were no significant differences between Tactile and Combined audio/tactile feedback.

The Wilcoxon signed-rank test showed that for Confirmations, Tactile and Combined audio/tactile feedback received a significantly higher number of votes. Error presentation through combined audio/tactile feedback results in significantly more votes than using audio or tactile feedback alone $(\mathrm{p} \leq 0.001)$. For progress updates and warnings, once again tactile and combined audio/tactile feedback received a significantly higher number of votes $(\mathrm{p} \leq 0.001)$.

These results suggest that the use of audio feedback is in general not the preferred modality but that perhaps, for information types requiring more attention-grabbing features, combining audio and tactile feedback is effective.

\subsection{Parameters}

The next step in the analysis was to investigate the effects of different design parameters on the choice of feedback for each information type. The average number of votes for each design 
parameter and information type is shown in Figure 5. Each of the four information types produced quite different results in terms of preferred parameters. For Confirmations, the use of rhythm was preferred the most with 22 votes while for Errors, both texture and tempo were chosen the most with 12 and 18 votes respectively. In terms of Progress updates, the votes are fairly spread out but location and duration received the highest number of votes. Likewise, Warnings presented using texture and tempo received a high number of votes (11 and 19).

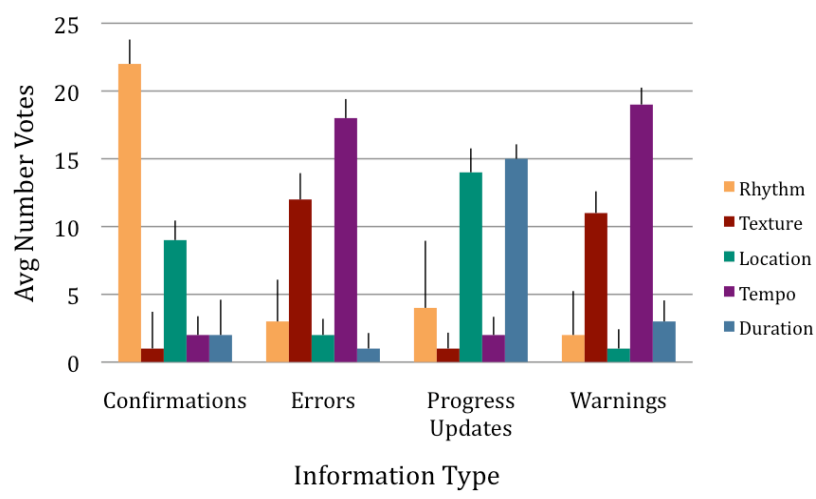

Figure 5: Average number of votes for each parameter and information type $(\max 36)$.

Analysis of the number of votes for each specific parameter and information type using a Friedman test showed a significant difference for parameter type $(\mathrm{Q}=9.69, \mathrm{df}=4, \mathrm{p}<0.05)$ and for information type $(\mathrm{Q}=18.6, \mathrm{df}=3, \mathrm{p}<0.001)$. Wilcoxon signed-rank tests with Bonferroni correction significance levels showed that there were significantly more votes for rhythm as the most important parameter when presenting Confirmation information $(\mathrm{Z}=22.37, \mathrm{df}=4, \mathrm{p} \leq 0.001)$. The analysis also showed that there were significantly more votes for texture $(Z=3.16$, df $=4, p=$ $0.034)$ than rhythm, location and duration when presenting error and warning information. The number of votes for spatial location were significantly higher than texture, tempo and duration when presenting confirmation information and significantly higher than rhythm, texture and tempo in progress updates $(\mathrm{Z}=4.13, \mathrm{df}=4, \mathrm{p}$ $=0.0065)$. The tempo parameter produced significantly more votes in error and warning information than rhythm, location and duration $(Z=7.44, d f=4, p=0.002)$. Duration produced significantly more votes than rhythm, texture and tempo in progress updates but no other significant differences $(Z=2.19, \mathrm{df}=4, \mathrm{p}=0.058)$.

These results indicate that for different types of information, different parameters in audio, tactile and combined audio/tactile feedback are ranked significantly higher than others. For instance, rhythm plays a big role in the presentation of confirmations but not in other types of information presentation.

\subsection{Individual Parameter Designs}

\subsubsection{Rhythm}

The average number of votes for each of the three different rhythms used is shown in Figure 6. The 4-beat rhythm was preferred for confirmations, while both the 2 and 4-beat rhythms were chosen for errors. For progress updates and warnings, the 4 and 6-beat rhythms were voted for most often.

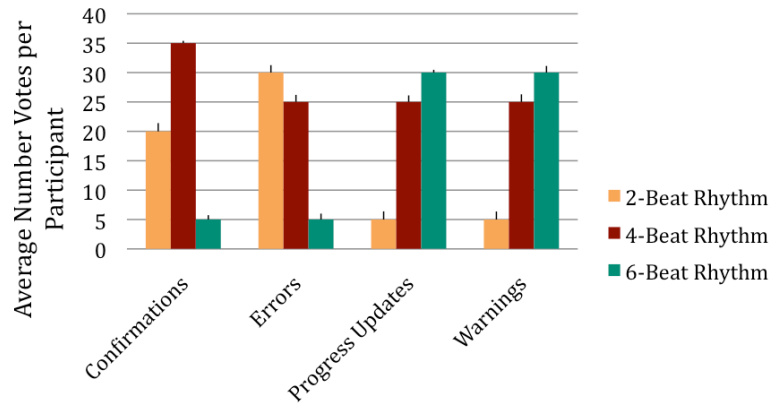

Information Type

Figure 6: Average number of votes for each rhythm design and each information type $(\max 60)$.

Analysis of the mean number of votes, comparing effects of information type and individual parameter design, showed a significant difference for rhythm type $(\mathrm{Q}=9.77, \mathrm{df}=2, \mathrm{p}<0.01)$ and for information type $(\mathrm{Q}=8.47, \mathrm{df}=3, \mathrm{p}<0.05)$. Bonferroni corrected post hoc Wilcoxon signed-rank tests showed that the 2-beat rhythm received significantly more votes for confirmations and errors than the 6-beat rhythm $(Z=7.79, \mathrm{df}=2, \mathrm{p}<0.001)$. The 4-beat rhythm also received significantly more votes than the 6-beat rhythm for confirmation information $(Z=15.92, \mathrm{df}=2, \mathrm{p}<0.0001)$. The 6-beat rhythm received significantly more votes than the 2beat rhythm when used to present warning and progress information $(Z=17.2, \mathrm{df}=2, \mathrm{p}<0.0001)$.

These results suggest that the 2-beat rhythm is preferred for presentation of confirmation and error information. Whereas the 6beat rhythm ranks higher when used to present progress updates and warnings.

\subsubsection{Texture}

The average number of votes for each texture and information type are shown in Figure 7. Confirmations presented using a smooth texture were voted for most often while a very rough texture was voted for most often when presenting errors and warnings. Progress updates using a smooth texture received 25 votes, 20 votes for medium rough textures and 15 votes for the roughest texture.

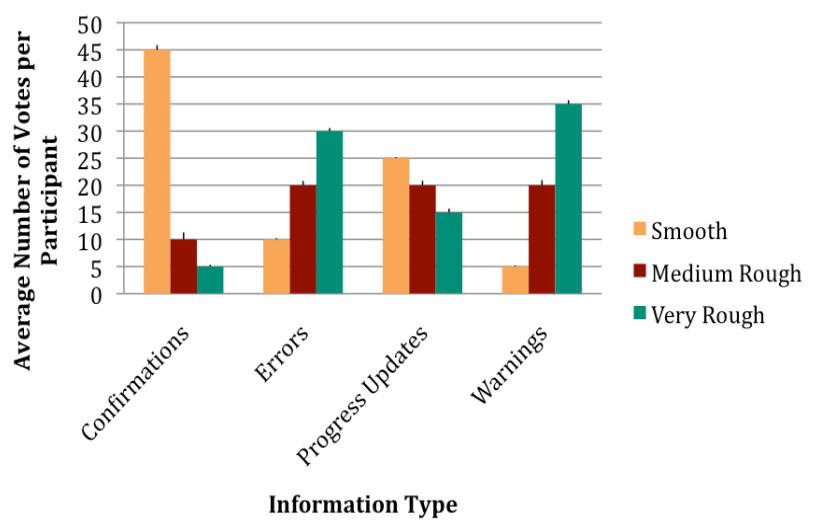

Figure 7: Average number of votes for each texture design and information type $(\max 60)$. 
Analysis of these data and all other parameter design data was completed using the same methods as above. The Friedman test on the texture data showed a significant difference for texture type $(\mathrm{Q}=36.05, \mathrm{df}=2, \mathrm{p}<0.001)$ and for information type $(\mathrm{Q}=27.5$, $\mathrm{df}=3, \mathrm{p}<0.001)$. Bonferroni corrected post hoc Wilcoxon signedrank tests showed that the smooth textured feedback produced significantly more votes than the medium rough and very rough textures when used to present confirmation information $(\mathrm{Z}=3.12$, $\mathrm{df}=2, \mathrm{p}=0.03)$. The very rough textured feedback received significantly more votes when used to present error and warning information compared to smooth and medium textured feedback $(\mathrm{Z}=3.41, \mathrm{df}=2, \mathrm{p}=0.005)$. There were no other significant differences.

It appears as though using smooth textures as opposed to rough textures is preferable for confirmation information and also slightly more preferred for progress information. At the other end of the scale, for information with perhaps more negative connotations or perceived urgency or importance such as errors and warnings, the very rough textured feedback ranks higher than smoother textures.

\subsubsection{Spatial Location}

The average number of votes for each spatial location is shown in Figure 8. Feedback presented to the center was most popular for confirmations with 35 votes. For errors and progress updates, feedback presented in a circular motion received the highest number of votes, 30 and 40 votes respectively. All spatial locations in warnings received 20 votes each.

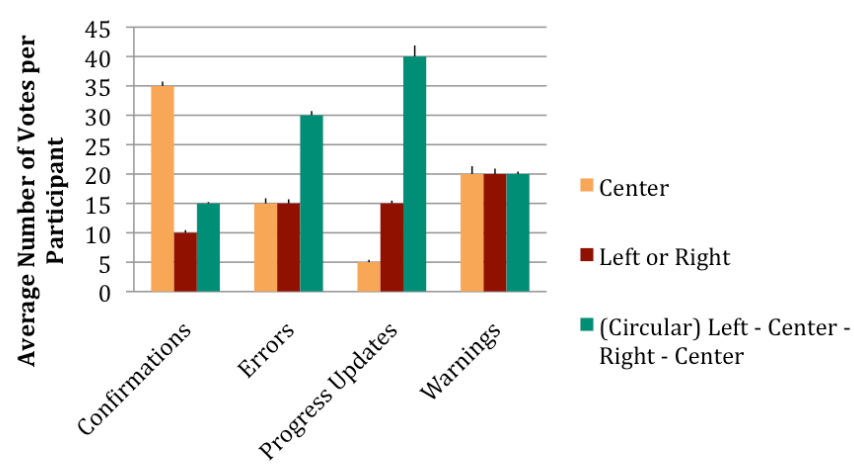

Information Type

Figure 8: Average number of votes for each spatial location design and information type $(\max 60)$.

In terms of spatial location, the Friedman test on the texture data showed a significant difference for each spatial location $(\mathrm{Q}=11.625, \mathrm{df}=2, \mathrm{p}<0.005)$ and information type $(\mathrm{Q}=13.45, \mathrm{df}=$ $3, \mathrm{p}<0.005)$. Bonferroni corrected post hoc Wilcoxon signed-rank tests showed that a central location when presenting confirmation information results in significantly more votes than using a left-toright motion or circular motion $(Z=9.78, \mathrm{df}=2, \mathrm{p} \leq 0.001)$. However, using a circular motion with progress updates produces significantly more votes than the other spatial locations $(Z=5.94$, df $=2, \mathrm{p}=0.05$ ).

\subsubsection{Tempo/Rate}

The average number of votes for each tempo/rate is shown in Figure 9. The slowest tempo was chosen most often for confirmations (40 votes). The fastest tempo was chosen most often for errors and warnings ( 30 votes) while all tempos received the same number of votes in terms of progress update presentation.

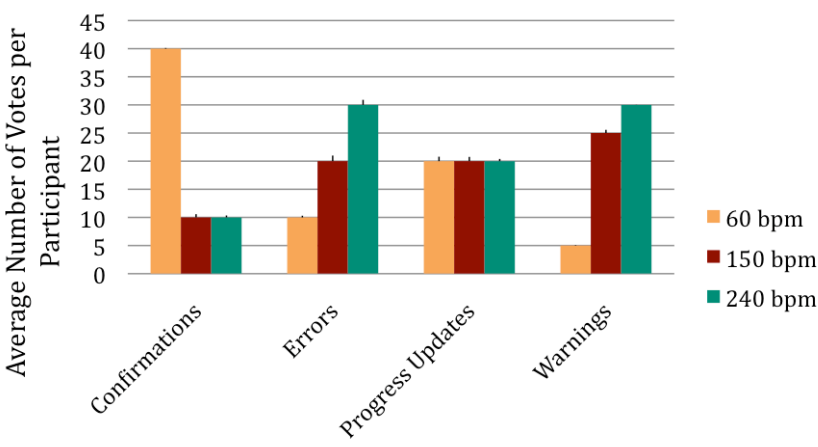

Information Type

Figure 9: Average number of votes for each tempo/rate and information type $(\max 60)$.

In terms of confirmation information, the Friedman test on the texture data showed a significant difference for each tempo $(\mathrm{Q}=$ $6.57, \mathrm{df}=2, \mathrm{p}<0.05)$ and for information type $(\mathrm{Q}=7.98, \mathrm{df}=3$, $\mathrm{p}<0.05)$. Bonferroni corrected post hoc Wilcoxon signed-rank tests showed that feedback presented at a tempo of 60 beats per minute is chosen significantly more often than the faster tempos $(\mathrm{Z}=6.23, \mathrm{df}=2, \mathrm{p}=0.0022$ ). For both warning and error information the fastest tempo of 240 beats per minute is chosen significantly more often than the slower tempos $(Z=6.97, \mathrm{df}=2$, $\mathrm{p}=0.006$ ). The medium tempo of 150 beats per minute was also chosen significantly more often than the slower tempo of 60 beats per minute for warning information $(\mathrm{Z}=5.86, \mathrm{df}=2, \mathrm{p}=0.0021)$. There were no other significant differences.

As with the results from the analysis of texture, it appears as though information with perhaps greater perceived urgency is most effectively mapped to faster tempos whereas simple confirmations are more congruent with a slower tempo.

\subsubsection{Duration}

The average number of votes for each duration and information type is shown in Figure 10. For confirmations, as the duration increases the number of votes decreases e.g. 25 votes for $100 \mathrm{~ms}$, 20 votes for 1 second and 15 votes for 2 seconds. For errors and progress updates, a duration of 2 seconds was voted for on average 35 times out of 60 . The $500 \mathrm{~ms}$ duration received zero votes when used to present progress updates. Warnings presented for 1 second received the highest number of votes (25) while shorter warnings received 20 votes and longer warnings only received 15 votes. 


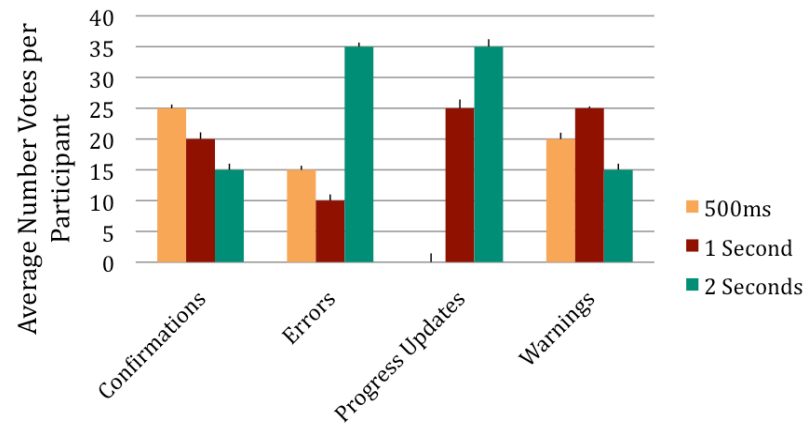

Information Type

Figure 10: Average number of votes for each duration and information type $(\max 60)$.

Analysis of the final parameter design using the Friedman test showed a significant difference for duration $(\mathrm{Q}=11.59, \mathrm{df}=2, \mathrm{p}$ $<0.005)$ and information type $(\mathrm{Q}=21.76$, df $=3, \mathrm{p}<0.001)$. Bonferroni corrected post hoc Wilcoxon signed-rank tests showed that showed that a duration of 2 seconds when presenting error and progress information is chosen significantly more often than the shorter durations $(\mathrm{Z}=3.46, \mathrm{df}=2, \mathrm{p}=0.0024)$. There were no other significant differences.

It is possible that longer durations are preferred for error information because shorter stimuli may be more easily missed and given that error information tends to be important to the user, a longer duration decreases the likelihood of an error going unnoticed. As for progress information, a longer duration may suggest that more progress has been made.

\section{DISCUSSION}

The experiment revealed several consistent ratings between participants and use case scenarios. The results are summarised below:

- Confirmations:

○ Modality: tactile, combined audio/tactile;

○ Parameters: rhythm, location;

- Parameter Design: short rhythms, and central spatial location.

- Errors:

- Modality: combined audio/tactile;

- Parameters: texture, tempo;

- Parameter Design: very rough texture, and fast tempo.

- Progress Updates:

- Modality: tactile, combined audio/tactile;

- Parameters: location, duration;

- Parameter Design: circular motion, and long duration.

- Warnings:

○ Modality: tactile or combined audio/tactile;

- Parameters: texture, tempo;

- Parameter Design: very rough texture and fast tempo.
Hypothesis 1 can be accepted given that significant differences were found in the data for each information type with very little variation showing that participants can consistently map different information to different audio and tactile feedback. The results show that different parameter designs play greater roles than others in mappings between information types and audio or tactile feedback. For example, short rhythms are preferred for confirmations while longer rhythms are preferred when used to present progress updates.

Hypothesis 2 can also be accepted because tactile feedback was highest ranked when used to present confirmations and progress updates while combined audio/tactile feedback ranked highest for errors and warnings. Information presentation through audio feedback was rarely chosen by participants. This is an important finding given that most current commercial devices use basic audio feedback to provide information about incoming messages etc. These devices would be better served by presenting information using the tactile modality and then in more extreme situations where attracting user attention is crucial, fade in audio feedback alongside the tactile feedback.

\section{CONCLUSIONS}

This paper focused on how congruent sets of audio and tactile feedback can be created and mapped to specific information types. A paired-comparison experiment was conducted revealing relationships between information mappings and individual amodal parameters in the audio and tactile modalities such as rhythm, texture, tempo, spatial location and duration.

For the majority of the time it was established that audio feedback does not rank highly compared to tactile feedback and combined audio/tactile feedback in terms of user preference for information such as confirmations, errors, progress updates and warnings presented by mobile devices. However, in certain situations tactile feedback may not always be appropriate e.g. vibrations can often go unnoticed if the device is not in contact with the user's skin. In this case, audio feedback would be beneficial.

When mapped to each of the different individual information types, certain different parameters of the audio and tactile feedback were ranked considerably higher than others. For confirmations, the most important parameters are rhythm and location. More specifically, short 2-beat rhythms and simple central locations. However, for progress updates, a circular pattern created by altering the spatial location of the feedback was preferred. Some participants commented that this spatial pattern mimicked visual icons such as spinners and progress bars. For both errors and warnings, which could be considered the most important types of information for users, fast tempos and rough textures ranked highly. This is most likely because fast and rough textured stimuli are more attention grabbing than those with smooth textures and slow tempos, which is vital to ensure that important information does not go unnoticed. The duration of crossmodal feedback did not perform as expected. For most types of information the duration did not play an important role. Nevertheless, duration is crucial for other reasons. The feedback must have a long enough duration for users to be able to distinguish the features of the feedback

In the future, ecological considerations will also be addressed as the results may change when parameters are combined or are redesigned to represent real-world objects as opposed to abstract messages from an application. Increasing the number of parameters may also provide useful results. There are many more pa- 
rameters available in the audio modality than in the tactile modality such as pitch. Perhaps by employing these additional parameters the ranking results of the audio modality may improve. Furthermore, a larger number of different types of application will be examined in addition to the three hypothetical applications used in this experiment.

This paper has presented practical implementation details and guidelines based on the results from our information mapping experiment focusing on the appropriateness of the audio and tactile modalities for different types of information presentation and then, more specifically, focusing on individual design parameters. Drawing on the results, we argue that a combination of audio parameters or tactile parameters can be produced which allow the same type of information to be derived through touch and sound with an intuitive match to the content of the message.

\section{ACKNOWLEDGMENTS}

This work is funded by the Finnish Funding Agency for Technology and Innovation (decision 40377/08).

\section{REFERENCES}

[1] Bernsen, N. O., "Modality Theory in Support of Multimodal Interface Design," ERCIM Workshop on Multimodal HumanComputer Interaction, pp. 27 - 44, 1993.

[2] Blattner, M. M., Sumikawa, D. A., and Greenberg, R. M., "Earcons and Icons: Their Structure and Common Design Principles," Human Computer Interaction, vol. 4(1), pp. 11 44, 1989.

[3] Brewster, S. A., Wright, P. C., and Edwards, A. D. N. Experimentally Derived Guidelines for the Creation of Earcons. In Proc HCI '95, Springer (1995), pp. 155 - 159.

[4] Brown, L. M. and Brewster, S. A. Multidimensional Tactons for Non-Visual Information Display in Mobile Devices. In Proc MobileHCI '06, ACM Press (2006), pp. 231 - 238.

[5] Chang, A. and O'Sullivan, C. Audio-haptic feedback in mobile phones. In Proc CHI '05 extended abstracts on Human factors in computing systems, ACM Press (2005).

[6] Edworthy, J., Hellier, E. J., and Hards, R., "The Semantic Associations of Acoustic Parameters Commonly Used in the Design of Auditory Information and Warning Signals," Ergonomics, vol. 38(11), pp. 2341 - 2361, 1995.

[7] Enriquez, M., MacLean, K., and Chita, C. Haptic Phenomes: Basic Building Blocks of Haptic Communication. In Proc 8th International Conference on Multimodal Interfaces (ICMI'06), IEEE (2006), pp. 302 - 309.

[8] Gaver, W., "Auditory Icons: Using Sound in Computer Interfaces," ACM SIGCHI Bulletin, vol. 19(1), p. 74, 1987.
[9] Geldard, F. A., "Some Neglected Possibilities of Communication," Science, vol. 131(3413), pp. 1583 - 1588, 1960.

[10] Hoggan, E. and Brewster, S. A. Designing audio and tactile crossmodal icons for mobile devices. In Proc Proceedings of the 9th international conference on Multimodal interfaces, ACM Press (2007), pp. 162 - 169.

[11] Hoggan, E. and Brewster, S. A. New Parameters for Tacton Design. In Proc Vol II CHI 2007, ACM Press (2007), pp. $2417-2422$.

[12] Koskinen, E., Kaaresoja, T., and Laitinen, P. Feel-Good Touch: Finding the Most Pleasant Tactile Feedback for a Mobile Touch Screen Button. In Proc ICMI 2008, ACM Press (2008), pp. 297 - 304.

[13] Lewkowicz, D. J., "Development of Intersensory Perception in Human Infants," in The Development of Intersensory Perception: Comparative Perspectives. Eds. D. J. Lewkowicz and R. Lickliter. Hillsdale, N.J.: Erlbaum, 1994, pp. 165 203.

[14] MacLean, K. and Enriquez, M. Perceptual Design of Haptic Icons. In Proc Eurohaptics, 2003, pp. 351 - 363.

[15] McGookin, D. and Brewster, S. A., "Understanding Concurrent Earcons: Applying Auditory Scene Analysis Principles to Concurrent Earcon Recognition," ACM Transactions on Applied Perception, vol. 1, pp. 130 - 155, 2004.

[16] Mortimer, B., Zets, G., and Cholewiak, R. W., "Vibrotactile Transduction and Transducers," The Journal of the Acoustic Society of America, vol. 121, pp. 2970 - 2977, 2007.

[17] Palomäki, H. Meanings Conveyed by Simple Auditory Rhythms. In Proc ICAD'06, 2006.

[18] Patterson, R. D., Guidelines for Auditory Warning Systems on Civil Aircraft, C.A.A. Paper 82017, C. A. Authority (1982).

[19] Poupyrev, I. and Maruyama, S. Tactile Interfaces for Small Touch Screens. In Proc 16th Annual ACM Symposium on User Interface Software and Technology, ACM Press (2003), pp. 217 - 220.

[20] Rovers, A. F. and van Essen, H. A. Using Haptic Feedback in Everyday Products. In Proc EuroHaptics, Springer-Verlag (2006), pp. 447 - 453.

[21] Shneiderman, B., Designing the User Interface. Reading, MA: Addison-Wesley, 1998.

[22] van Erp, J. B. Tactile Navigation Display. In Proc First international Workshop on Haptic Human - Computer Interaction, LNCS (2001), pp. 165 - 173.

[23] van Erp, J. B. and van Veen, H. A. H. C. Vibro-tactile information presentation in automobiles. In Proc EuroHaptics 2001, Springer Verlag (2001), pp. 99 - 104. 